\title{
EEG as a Controller for Psychedelic Visual Music in an Immersive Dome Environment
}

\author{
Jonathan Weinel, Stuart Cunningham, Nathan Roberts, Shaun Roberts and Darryl Griffiths \\ Creative \& Applied Research for the Digital Society (CARDS) \\ Glyndŵr University Plas Coch Campus, Mold Road, \\ Wrexham, LL11 2AW \\ North Wales, UK
}

\section{INTRODUCTION}

Psych Dome (Weinel 2013a) is a short interactive piece of visual music first presented in an immersive 'full dome' environment that forms part of the authors' on-going research regarding Altered States of Consciousness (ASC) as a basis for the design of computer-based artworks.

Following the Activation, Input, Modulation (AIM) model of consciousness provided by Hobson (2002), Psych Dome seeks to represent the visual material that may be generated internally when human consciousness is situated at the appropriate point on the Input axis. Specifically the 'form constant' visual patterns of hallucination discussed by Klüver (1966), such as the funnel dot patterns that may be perceived in hallucinatory experiences, are used as a basis for the design of visual materials within the work (see Figure 1). These are accompanied by suitable corresponding sonic materials, using approaches Weinel has developed through the course of his previous work composing electroacoustic music based on ASC (Weinel 2013b). The work can therefore be seen to extend the canon of other similar works in visual music that have also explored visual forms derived from hallucinations and experiences of the 'inner eye', such as Jordan Belson's films (Wees 1992).

In order to conceptually link the artwork interactively to brain activity, a consumer-grade electroencephalograph (EEG) headset is used as a control device. The headset is seen as a means to provide the constant change that characterises ASC (Cardeña \& Winkelman 2011). As part of the first presentation of Psych Dome, a pilot study was also conducted which enables us to consider the efficacy of the NeuroSky MindWave device as a controller for interactive artworks of this type.

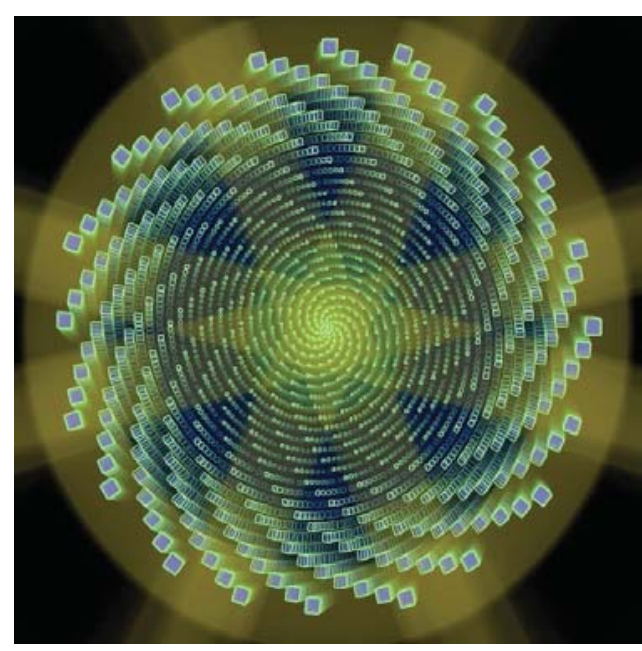

Figure 1: Excerpt from Psych Dome showing visual material based on hallucinatory form constants. (C) 2013 Weinel et al.

\section{SYSTEM AND COMPOSITIONAL DESIGN}

Figure 2 shows the system design used for Psych Dome; signals from the MindWave are received wirelessly and translated to OSC using Trent Brooks BrainWaveOSC software (2013). These are then used as control signals for a Max/MSP patch which generates the sound, and a Processing patch that generates the visual materials.

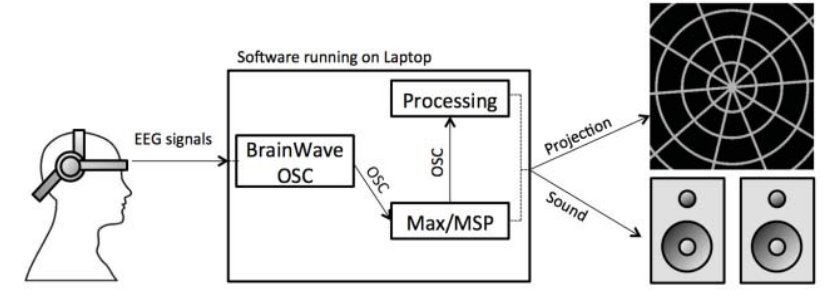

Figure 2: Psych Dome System Diagram. (c) 2013 Weinel et al. 
The composition follows a pre-determined structure, where certain sonic and visual materials such as animated spirals or tunnel patterns occur at fixed points in time, over a 100 second duration. Within this structure, control signals from the MindWave such as the interpreted attention and meditation values the headset provides are used to affect parameters of the sounds and graphics; hence the colours, size and form of shapes change in each presentation, as do various properties such as the frequency of sounds which are heard.

\section{USER EXPERIENCE}

From preliminary testing, the expectation was that the EEG headset would provide limited volitional 'active' control over the artwork, but could provide the participants with a sense of non-volitional 'passive' connection to the artwork. The pilot study investigated how tangible this sense of connection was by comparing the real EEG signal with a nonreal-time placebo signal. The results of this pilot study suggest that the participants could not tell a significant difference between the real and placebo EEG signals. This therefore suggests that while the device conceptually links brain activity to the generation of psychedelic visuals - a concept that may be viewed as interesting in and of itself for this work - further work would be needed to establish a more tangible sense of connection between the participant and the artwork. As has been suggested, this improvement may be provided by addressing the three central challenges of BrainComputer Interfaces (BCl): improving the quality of the $\mathrm{BCl}$, providing an improved training phase, or improving the implementation of the signals (Miranda, Durrant \& Anders 2008).

\section{SUMMARY}

We propose that Psych Dome is an example of a small scale BCI/ASC artwork. The work establishes the concept of an interactive piece of visual music based on the form of the visual patterns typically perceived in hallucinations. It also explores the use of a consumer-grade $\mathrm{BCl}$ as a controller for this, but demonstrates that while conceptually appealing, a less subtle sense of connection may be provided through other means.

\section{REFERENCES}

Brooks, T., and Khut, G. (2013) BrainwaveOSC [software application]. Available online: https://github.com/trentbrooks/BrainWaveOSC (retrieved 10 October 2013).

Cardeña, E., and Winkelman, M.J. (eds.) (2011). Altering Consciousness: Multidisciplinary Perspectives, Volume 1: History, Culture and the Humanities. Praeger. p.8.

Hobson, A. J. (2002) The Dream Drugstore: Chemically Altered States of Consciousness. MIT Press. pp.44-45.

Klüver, H. (1966) Mescal \& Mechanisms of Hallucinations. University of Chicago Press. p.66.

Miranda, E., Durrant, S., and Anders, T. (2008) Toward Brain-Computer Music Interfaces: Progress and Challenges. Proceedings of the International Symposium on Applied Sciences in Biomedical and Communication Technologies (ISABEL2008), Aalborg, Denmark.

Wees, W. C. (1992) Making Films for the Inner Eye: Jordan Belson, James Whitney, Paul Sharits. In Light Moving in Time: Studies in the Visual Aesthetics of Avant-garde Film. University of California Press.

Weinel, J. (2013a) Psych Dome [interactive audiovisual artwork]. First presentation at William Aston Hall, 16 October 2013, Glyndŵr University, Wales. Demonstration videos available online: https://vimeo.com/77236955 and https://vimeo.com/78153713 (retrieved 21 October 2013).

Weinel, J. (2013b) Visual Patterns of Hallucination as a Basis for Sonic Arts Composition. ACM Proceedings of Audio Mostly 2013. Luleå University of Technology, Piteå, Sweden. 